Informal Report

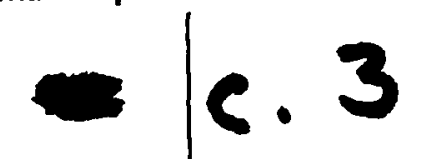

UC-20

Reporting Date: February 1975 Issued: October 1975

CIC-14 REPORT COLLECTION

REPRODUCTION

COPY

\title{
Performance of \\ Structured Laser Fusion Pellets
}

by

R. J. Mason
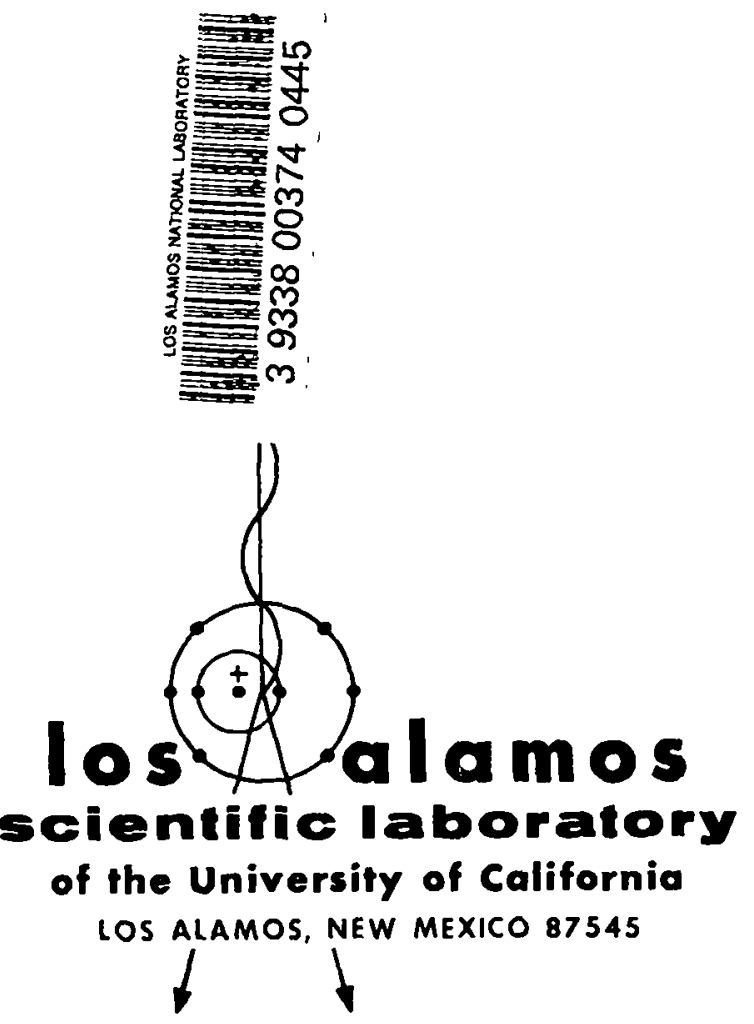

An Affirmative Action/Equal Opportunity Employer 
In the interest of prompt distribution, this report was not edited by the Technical Information staff.

Printed in the Unitod Staten of Amorica. Available trom Netional Technical Iniormation Service

US Department of Commerce

5285 Port Royal Road

Springtiold, VA 22151

Price: Printed Copy $\$ 4.00$ Microtiche $\$ 2.25$

This report was prepared as an eccount of work spontored

nor the Unleed States Enetry Research and Development Ad.

minintration. nor any of their employere, nor any of theis con-

tractors, subcontrectors or their empioyes. makes any

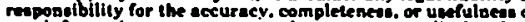

anvi Intormation. apparatud, produet, or procenos disclomed, or

represents that its use would not infrince privaleily owned 
PERFORMANCE OF STRUCTURED LASER FUSION PELLETS

by

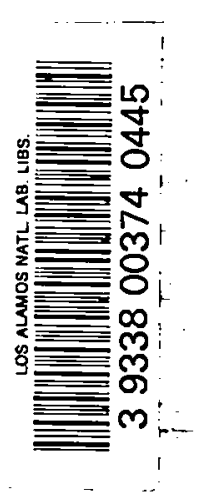

R. J. Mason

ABSTRACT

Results are presented from an extensive study of the hydroburn response of bare DT shells, inert shells, and DT-filled microballoons to ramp-like, Gaussian, and square laser pulses. Scaling laws are determined for the best pulse energy and time scale for shells, as functions of their mass, aspect ratio and inftlal density. The advantages of DT Ice over DT gas in the mfcroballoons are demonstrated. An equivalence rule is established between linear ramp pulses and Gaussians, and a connection is drawn between the ramps and square pulses. Projections are made, via the scaling rules, to breakeven-plus pellet designs. The scaling rules are used to recommend pulses for pellets recently imploded at LASL and KMSP. The calculated performance of these targets 1s shown to agree, after adjustments, with the laboratory findings. Attention 1s drawn to practical problem areas in structured pellet design.

\section{INTRODUCTION}

Laser-driven compression has been proposed as a means for the extraction of breakeven ylelds from bare DT spheres and shells. ${ }^{1-3}$ In recently reported laboratory experiments, ${ }^{4-8}$ however, the lasers have been fired at glass microballoons contalning the DT "fuel" as a gas. At LASL, one beam of $1.06 \mu \mathrm{m}$ light was delivered to a "ball and disk" target ${ }^{4,6}$ in a tuned sequence of Gaussian pulses of up to $50 \mathrm{~J}$. The purpose of the disk was to isotropize the energy deposition and implosive dynamics. At KMSF, two beams with a mirror-lens system to isotropize were delivered to the microballoons in ramp-like and square pulses. $5,7,8$

This report presents the results of computer simulations, which were performed: (a) to provide us with a general understanding of the microballoon Implosion phenomenology, (b) to help us match these targets to avallable laser pulses, and (c) to determine the scaling requirements for breakeven-plus configurations.

Under laser irradfation electrons near the surface of the shell are strongly heated, gener- ating a non-1inear thermal wave which penetrates the materlal below, causing it to stream away. The full mass of material which blows off is termed the "ablator". The reaction force to this ablation drives the remaining shell material towards the center, compressing it by shocks and convergence. This inner layer is termed a "pusher" in that it pushes the DT fuel within up to high densities. Also it is called a "tamper", since it subsequently provides inertial confinement of the burning fuel. ${ }^{9-11}$ We have found ${ }^{12}$ that the ablator should be preferably of low $Z$ material to mintmize the generation and reabsorption bremsstrahlung, Iine - and recombination radiation. On the other hand, we will show that the pusher should be of high $\mathrm{Z}$ to maximize the fuel compressions achieved. This means that composite shells, of, say, gold with an outer layer of beryllium, may ultimately be required. However, to simplify our task, the bulk of the present work has been restricted to homogeneous shell performance, with caveats, as to possible composite shell advantages, added where 
approprlate. The early shocks through the pusher should be weak to minimize hydrodynamlc preheat, 10 while the final shocks should be strong to provide a high fuel temperature for 1gnition, ${ }^{9}$ so pulse shaping is desirable, as with bare DT spheres. $1,2,10$ The shells can conveniently contain the DT as a gas, but it will be better to freeze it to the inner surface of the pusher, since this delays the production of significant back-pressure in the fuel, allowing for its greater compression.

To optimally match current targets and pulses we need to know the characteristic energles and time scales that can produce high compression, and the performance trends with shell mass, aspect ratio and initial density. An understanding of the dependence on the fuel parameters is also desirable, as is a feeling for the sensitivity to the detailed pulse profile.

Finally, comparison between theoretical expectations and experimental results is necessary to "normalize" our thinking, and to direct future efforts.

In Sec. II, we discuss the computer codes used in this study. In Sec. III, we develop scaling rules for ablatively imploded, simple structured targets. Section IV uses these rules to suggest breakeven-plus designs. In Sec. V, we analyze recent low energy experiments, and compare thel results with predictions from our simulations. Finally, Sec. VI discusses problem areas revealed, in part, by this comparison of theory and experiment.

\section{THE SIMULATION CODE}

Most of the simulations were performed with our one-dimenstonal, "3-T" Lagranglan hydro-burn code. It allows for separate Ion, electron and radiation temperatures, It accounts for electron and ion conduction and classical coupling between the electron and ton temperature fields. It can calculate energy exchange through bremsetrahlung and inversebremsstrahlung, and single group flux-limited radiation diffusion by Rosseland mean opacities. The code calculates the energy output from $D-T$ and $D-D$ reactions, following the transport and redeposition of $\alpha$-particle and neutron energy by the "Sn" method of Carlson. It uses tabular Fermi-Thomas-Dirac equations of state, including the effects of Ferm1. degeneracy on the pressures, Internal energies and opacities of the pellet materials.

A detailed discussion of the code is gtven in Ref. 9 with support from Ref. 10. To clarify the present calculations we reiterate the following:

(a) No real viscosity is employed. We use an artificial viscosity of the form

$Q_{j+1 / 2}= \begin{cases}2 \rho_{j+1 / 2}\left(u_{j+1}-u_{j}\right)^{2} & \dot{v}<0 \text { (compression) } \\ 0 & \dot{v}>0 \text { (expansion), }\end{cases}$

In whIch $\rho_{j+1 / 2}$ is the zone density at its center, and $u_{j+1}$ and $u_{j}$ are the velocities of the surroundIng zone boundarles. (b) The laser energy is deposited in the electrons by Inverse-bremsstrahlung up to the critical surface. Total anomalous absorption of any remaining energy is assumed and accounted for by dumping it into the first zone where the density exceeds the critical value.

(c) For wost of the runs the electron thermal flow was constratned to the classical 1 imit ${ }^{10,13}$

$$
\left.q\right|_{\max }=\beta \frac{\mathrm{n} \overline{\mathrm{c}}}{4}\left(3 K \mathrm{e}_{\mathrm{e}} / 2\right)
$$

with $\bar{c}=\left(8 \mathrm{KT} \mathrm{e}^{/ \pi m_{e}}\right)^{1 / 2}$ and $\beta=1.0$. In fact, we found little sensitivity to $\beta$ for $\beta \cong 0.3$. In scrutinizing the KMSF experiments, runs were made .rth $B$ as low as 0.006 .

The code does no superthermal electron transport. It also does no line or recombination radiation transport, so it misses preheat effects that afflict pellets with high $z$ ablators. ${ }^{12}$ Futhermore, for computational speed in the many calculations, even the bremsstrahlung generation was suppressed (with $A_{\text {er }}$ set to zero in Ref. 9, Eq. (B2)) in most of the runs. The consequences of these defictencies are discussed at appropriate points in the text, with reference to comparable results from the alternate non-equilibrium, multigroup photonics code, also detalled in Ref. 9.

\section{PELLET RESPONSE CHARACTERISTICS}

We first characterize the hydroburn response of a high aspect ratio, bare DT shell to a linear ramp pulse. We find the time scale $\tau$ and the energy $\varepsilon$ which give the best yfeld ratio $Y_{R}=Y / \varepsilon$. We characterize the separate dependencies on time scale and energy input. Then, we find the scaling to shells of other masses, aspect ratios, and 
DT ice shell
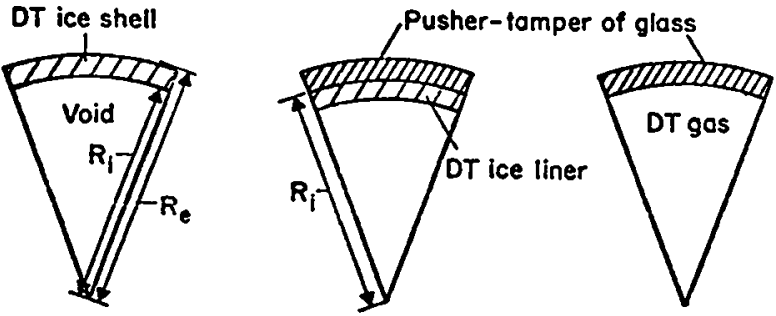

Fig. 1 Types of simple structured spherlcal laser fusion pellets.

higher $z$. This gfves the response of shells of 1ithium, plastic (CDT), glass, nickel and gold. Next, we relate the homogeneous shell results to the phenomenology that applies when DT 1ce or DT gas is in the shells. The types of targets considered are sketched in Fig. 1 . Finally, we show the hydrodynamic connections between 11 near ramps, Gaussians and square pulses, and the predicted advantages of prepulsing, by going to extended ramps of the form

$$
P=\partial E / \partial t=\frac{(p+1) \varepsilon}{\tau}(t / \tau)^{p} \quad p>0
$$

The wavelength is $1.06 \mu$ for all the calculations.

\section{A. $\tau$ and $\varepsilon$ Dependence}

To start we chose to study the implosion characteristics of a 7.5- $\mu \mathrm{g}$ DT shell of aspect ratio 32. A 7.5- $\mathrm{g}$ g mass was selected because previous1y we have discussed bare DT shell ${ }^{2}$ and spheres ${ }^{10}$ of this mass. Also, it turns out that this shell requires about one kilojoule of absorbed energy for optimal response. This is in line with optimistic expectations for the deliverable energy from lasers which should be available during the next few years. The high aspect ratio was chosen because it provides a good run-up distance for the shell wall prior to the central collapse, and because it was consistent with the ratio in recent LASL and KMSF experiments. We were well aware that an aspect ratio of 32 might prove to be too large for hydrodynamic stabtlity in ablative implosions. This, in part, motivated our interest in developing the scaling to lower aspect ratios, which is given in Sec. III $C$.

A matrix of more than 100 runs was made to extract the implosion characteristics of the DT shell as a function of $\tau$ and $\varepsilon$. Its initial density was $0.213 \mathrm{~g} / \mathrm{cm}^{3}$ (equi-molar DT). Its inner radius starts at $R_{i}=438 \mu \mathrm{m}$, and 1 ts outer radius is $\mathrm{R}_{\mathrm{e}}=452 \mu \mathrm{m}$, giving the aspect ratio, $\mathrm{R}_{\mathrm{a}}=$ $R_{e} / \Delta R=32, \Delta R=R_{e}-R_{i}$. With the linear ramp ( $P=1$ ) the best yleld ratlo, $Y_{R}=4.9 \%$ was obtained with $\tau=\tau^{*}=1.8$ ns and $\varepsilon=\varepsilon^{*}=1.2 \mathrm{kj}$. Such starred values will be termed the "optimal" pulse
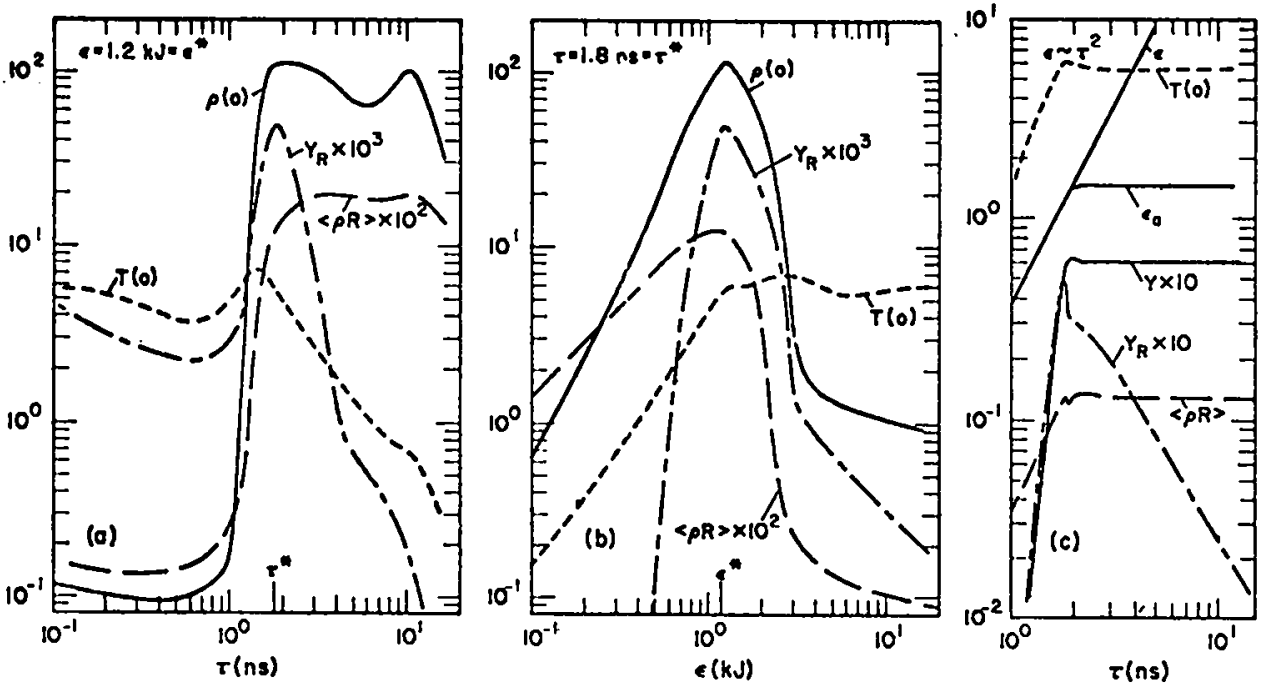

Fig. 2 DT shell response to changes in the ramp: (a) time scale $\tau$,

(b) energy $\varepsilon$, (c) energy and tfme scale together such that

$\varepsilon \sim \tau^{2}$. 
parameters.

F1gure 2 shows how shell response changes as one varies: (a) the time scale with $\varepsilon$ fixed at $\varepsilon^{*}$, (b) the energy with $\tau$ fixed at $\tau^{*}$, and (c) $\varepsilon$ and $\tau$ together but connected through the relation $\varepsilon \sim \tau^{2}$. As diagnostics for the simulations we record:

(i) the yield ratio, $Y_{R}=Y / \varepsilon$,

(i1) the maximum value of the integral $\int_{0}^{\mathrm{O}_{\mathrm{e} R}} \equiv\langle\rho \mathrm{R}\rangle$ in $\mathrm{g} / \mathrm{cm}^{2}$, as a measure of the useful compression for burn,

(11i) $\rho(0)$ in $8 / \mathrm{cm}^{3}$, the maximum central density achieved, and

(iv) $T(0)$ In keV, the central temperature at the time of the maxirum central density.

These units apply in all the figures of the paper, except as otherwise noted.

Looking carefully at the time scale dependence, we see from F1g. 2(a) that $\rho(0)$ drops precipitously for $\tau<0.7 \tau^{*}$. The thermal wave, which should be driving shocks ${ }^{2}$, 10 through the shell and Imploding $1 t$, Instead burns though the shell wall prior to it.s full central collapse. The pulse power runs too high. Simflarly, burnthrough occurs when, on the optimal scale $\tau^{*}$, too much energy, $\varepsilon \widetilde{>} 2.0 \varepsilon^{*}$, from Fig. $2(\mathrm{~b})$, is delivered. Good compressions are possible with the time scales up to $\tau=10 \tau^{*}$, but then $T(0) \mathrm{de}-$ clines as $\tau^{-1}$. When the longer time scales are used, the shell collapses too slowly to give a good conversion of kinetic energy to thermal energy at the pellet center.

Examining the energy dependence, we find that for energies below $\varepsilon^{*}$ the density drops rapidly as $\rho(0) \sim \varepsilon^{2}$, while the temperature and $\langle\rho R\rangle$ obey $T(0) \sim\langle\rho R>\sim \varepsilon$. There is not enough energy deIfvered to complete the ablabatic compression of the shell. Alternatively, for $\varepsilon>\varepsilon^{*}, T(0)$ remains nearly constant out to $10 \varepsilon^{*}$, but $\langle\rho R\rangle$ drops rapidly due to burn-through.

Figure 2(c) provides the most important insight. It shows that if $\tau$ is too long it is possIble to correct the mismatch by increasing $\varepsilon$. With the ramp pulses $\mathrm{Rq} .(1)$, the scaling $\varepsilon \sim \tau^{\mathrm{p}+1}$, here $\varepsilon \sim \tau^{2}$, keeps the pulse form optimal up to $t=\tau^{*}$, which is roughly when the central collapse occurs. Any additional energy is then simply wasted, arriving after the collapse, and reducing the yleld ratio by $Y_{R} \sim \varepsilon^{-1}$. In figure $2(c) \varepsilon_{a}$ is the energy in kilojoules absorbed up to the time of the central density maximum. It asymptotes at $1.2 \varepsilon^{*}$. The central temperature and $\langle\rho R\rangle$ are nearly constant for $\varepsilon \sim \tau^{2}>\tau^{* 2}$.

We see that: (a) the optimal $\tau$ and $\varepsilon$ values, which give the maximum $Y_{R}$ in DT shells, also give, very nearly, the maximum $\rho(0)$, and (b) $\varepsilon^{*}$ is the minimum energy that provides this $\rho(0)$.

Figure $2(c)$ suggests a simple way to tune the ramp pulses to a given pellet. It reduces the search from two parameters to one. The procedure is as follows: A sufficiently large $\tau$ is chosen. Then one varles $\varepsilon$ over a large range (in either simulations or laboratory experiment) unt1l the best $Y_{R}$ or $\rho(0)$ is obtained. Then one reduces $\varepsilon$ and $\tau$ together, with $\varepsilon \sim \tau^{p+1}$. The target performance should be constant until $\varepsilon$ drops below $\varepsilon^{*}$. In practice, with the small targets now in use at low energy this may select a time scale that is too short for the available laser system (say 100 to $180 \mathrm{ps}$ ). In this case, one can operate at longer $\tau$ with the approprlate $\varepsilon$ increase. This rule also applies with single Gaussian pulses, as shown in Section III F.

B. Mass Dependence

To check the mass dependeace of performance the $p=1, \varepsilon^{*}$ and $\tau^{*}$ values were found for $R_{a}=32 \mathrm{DT}$ shells of mass $0.4,4.4,7.5,58$, and $250 \mu \mathrm{g}$. The results appear in Fig. 3 . In addition to the properties recorded In Fig. 2, we give, as well: the optimal $\varepsilon^{*} / \mathrm{m}$ in $\mathrm{kJ} / \mu_{\mathrm{g}}$,

(1ii) the peak input power level $P=2 \varepsilon^{*} / \tau^{*}$ in $\mathrm{J} / \mathrm{ps}$,

(iv) the peak intensity of energy deposition at the critical surface $\mathrm{Q} \equiv \mathrm{P} / 4 \pi \mathrm{R}_{\mathrm{e}}^{2}$ in $\mathrm{W} / \mathrm{cm}^{2}$ ( the critical surface remains near the initial pellet edge radius under $1.06 \mu$ m illumination. 10 

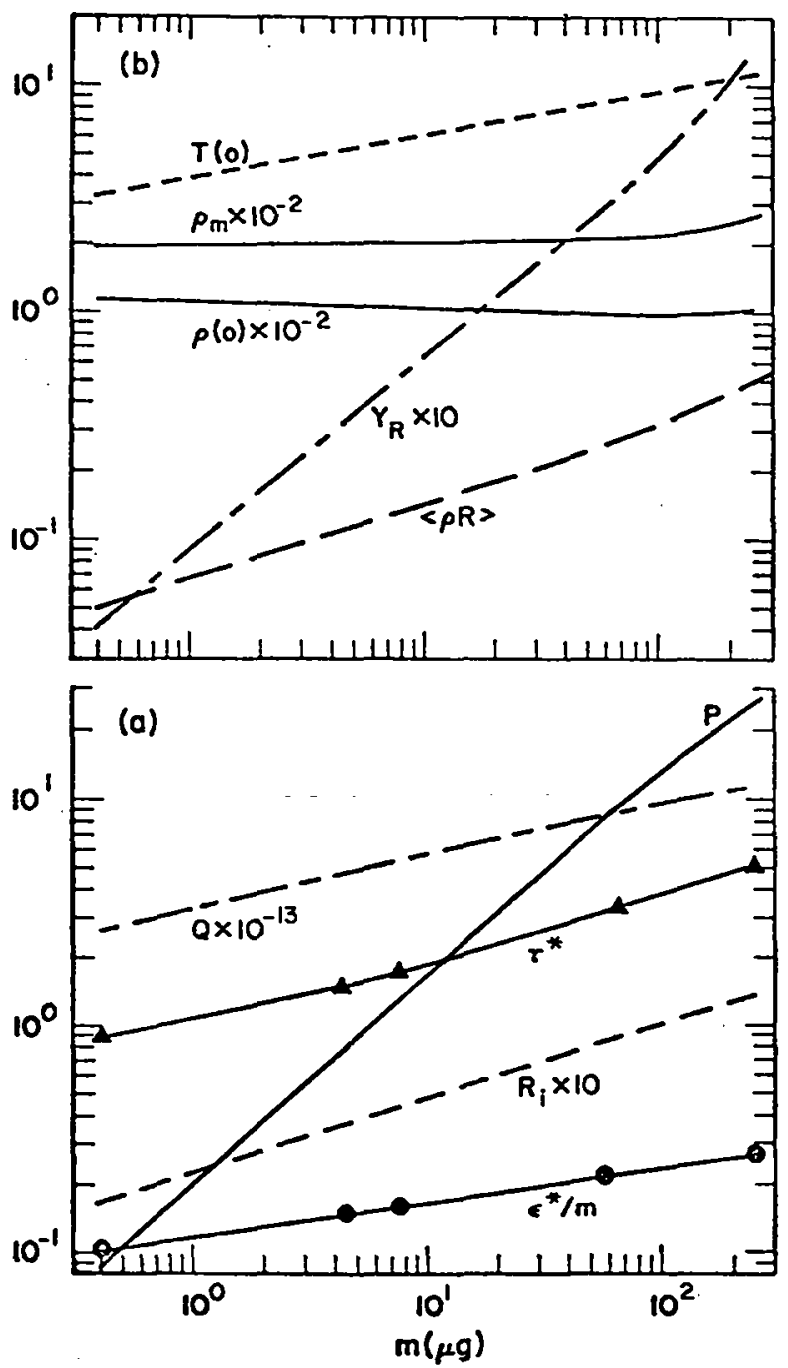

Fig. 3 Mass dependence of performance of the $\mathrm{R}_{\mathrm{a}}=$ 32, $\rho_{0}=0.213 \mathrm{~g} / \mathrm{cm}^{3}$ DT shell.

(v) $\rho_{\text {m }}$ in $\mathrm{g} / \mathrm{cm}^{3}$, the maximum density registered in the shell

when $\rho(0)$ is recorded.

Again, these units apply to the corresponding curves in all subsequent figures of the paper.

The main reault is that all the $<58-\mu \mathrm{g}$ shell implosions are self-similiar. The peak central and overall densities are independent of the mass. The central zone compresses 483 -fold from 0.21 to $100 \mathrm{~g} / \mathrm{cm}^{3}$.

To establish this self-similarity we note that, wh1le $\rho(0) \sim$ const., $\left\langle\rho R>\sim \mathrm{m}^{0.34}\right.$. Now an estimate of the shell mass that compresses is ${ }^{10}$

$$
\mathrm{m}_{0}=\frac{4 \pi}{3} \frac{\langle\rho R\rangle^{3}}{\rho(0)^{2}} 2 \sim\left(\mathrm{m}^{0.34}\right)^{3} \sim \mathrm{m} \text {, }
$$

i.e., a constant fraction $\mathrm{m}_{\mathrm{o}} / \mathrm{m}$ of the initial shell mass $m$ is compressed. At $7.5 \mu \mathrm{g} \mathrm{Eq.} \mathrm{(2)} \mathrm{gives} \mathrm{this}$ as $11 \%$. (A more accurate value for the compressed fraction requires a precise relation between $\rho(0)$ and $\langle\rho\rangle$, the peak average compressed shell density. Larger pellets require a longer pulse; $\tau^{*}$ scales roughly with $R_{1}$, i.e., $\tau^{*} \sim m^{0.27} \sim R_{i}^{0.82}$. Thus, the mean velocity of collapse is nearly constant

$$
\vec{v} \equiv R_{i} / \tau^{*} \sim R_{1}^{0.18} \sim m^{0.06} \text {. }
$$

The 7.5- $\mu \mathrm{g}$ shell collapses at $\bar{v}=2.4 \times 10^{7} \mathrm{~cm} / \mathrm{s}$. The energy needs scale roughiy with the mass, as is true with the fully optimal pulse, ${ }^{2}, 10$ but less energy must be delivered in the ramp pulses, and more mass ablates. The range for $E^{*} / m$ is from 0.1 to $0.25 \mathrm{~kJ} / \mathrm{\mu g}$ for the masses studied. Breakeven $\left(Y_{R}=1\right)$ is predicted for $m=190 \mu \mathrm{g}$ and $\varepsilon^{*}=$ $50 \mathrm{~kJ}$. This emphasizes the advantage gained with even this simple pulse shaping, since for a square pulse we find that $120 \mathrm{~kJ}$ is the minimum breakeven energy, and this requires an aspect ratio exceeding 250. Finally, note that the increase in $\varepsilon^{*} / m$ with the larger shell masses correlates with the higher central temperatures and higher collapse velocities

TABLE I

SCALING RULES FOR SHELLS:

\begin{tabular}{|c|c|c|c|}
\hline Property & $\underline{m(<58 \mu \delta)}$ & $R_{B}(225)$ & $D_{0}\left(<10 \mathrm{~s} / \mathrm{cm}^{3}\right)$ \\
\hline$\tau^{\star}$ & 0.27 & 0.56 & -0.37 \\
\hline$R_{1}$ & 0.33 & 0.44 & -0.33 \\
\hline$\varepsilon^{\star}$ & 1.14 & -0.38 & 0.50 \\
\hline $\mathbf{P}$ & 0.87 & -0.94 & 0.87 \\
\hline$Q$ & 0.21 & -1.59 & 1.53 \\
\hline$\langle P R\rangle$ & 0.34 & 0.84 & 0.76 \\
\hline$p(0), p_{\mathrm{m}}$ & 0.0 & 1.28 & $0.95,1.07$ \\
\hline$I(0)$ & 0.19 & -0.42 & 0.13 \\
\hline$y_{R}$ & 0.87 & 0.15 & - \\
\hline $\bar{\nabla}$ & 0.06 & -0.12 & 0.04 \\
\hline n。 & 1.00 & -0.04 & 0.38 \\
\hline$m_{0} v^{-2}$ & 1.12 & -0.28 & 0.46 \\
\hline
\end{tabular}
e. 8., $\tau^{*} \sim \mathrm{m}^{0.27}$ and $\tau^{\star} \sim p_{0}^{-0.37}$. 
required to achieve a maximum $\mathrm{X}_{\mathrm{R}}$ as in rises.

The optimal laser peak power $P$ runs from 0.09 $\mathrm{J} / \mathrm{ps}$ at $0.4 \mu \mathrm{g}$ to $25 \mathrm{~J} / \mathrm{ps}$ when $\mathrm{m}=250 \mu \mathrm{g}$, and $P \sim m^{0.87}$. This is reasonable since energy balance suggests that

$$
\begin{aligned}
& P \sim \rho(0) T(0) \bar{v} R^{2} \\
& \sim \text { const. } m^{0.19} \cdot m^{0.06}\left(m^{0.33}\right)^{2} \sim m^{0.91} .
\end{aligned}
$$

The Intensity $Q$ changes from $3 \times 10^{13} \mathrm{~W} / \mathrm{cm}^{2}$ to $1.4 \times 10^{14} \mathrm{~W} / \mathrm{cm}^{2}$, and $Q \sim \mathrm{m}^{0.21}$. This $1 \mathrm{~s}$ weaker than the $P$ dependence because of the increased surface area of the larger shells. Still Q rises for larger shells, which may lead to superthermal electron problems. With fully optimized pulses, 10 $Q$ declines with $\mathrm{m}$.

Table I. collects the various scaling rules discussed.

\section{Aspect Ratio}

Large aspect ratios demand lower peak intensities, but low aspect ratios may be required for stabllity. 13, 14, 15 Laboratory experiments will determine the practical limits on $R_{a}$. Figure 4 gives results of our one-dimensional calculations (which ignore the stability question) for aspect ratios from 61 to 1 (a sphere). Again, the pellet is a 7.5- $\mu_{8}$ DT shell, and $p=1$.

For $R_{a} \Upsilon 15$, the pellet properties and favored pulse tuning are smoothly dependent on $R_{a}$. The shell inner radius, $R_{f} \sim R_{a}^{0.44}$. The time scale tracks $R_{1} ; \tau \sim R_{a}^{0.56} \sim R_{1}^{1.27}$. Larger shells close somewhat more slowly, $\bar{v} \sim R_{a}^{-0.12}$.

The energy $\varepsilon^{*}$ drops by a factor of two, as $R_{a}$ goes from 8 to 50 ; $\varepsilon^{\star} \sim R_{a}^{-0.38}$. The laser power requirements are down for the larger shells, $P \sim R_{a}^{-0.44}$. Most Important, the peak intensity drops from both the declining $\varepsilon^{*}$ and the rising $\tau^{*}$ and $R_{1}$, so that $Q \sim R_{a}{ }^{-1.59}$.

The denstty and $\langle\rho R\rangle$ increase in such a way that Eq. (2) gives $m_{0} \sim R_{a}{ }^{-0.04}$; 1.e., the fraction of mass compressed is independent of the aspect ratio. On the other hand, the optimal tuning leads to cooler pellet centers, $T(0) \sim R_{a}{ }^{-0.42}$, which helps to explain the slow rise in $Y_{R}$ with $R_{a}$. One expects that 9

$$
\mathrm{Y}_{\mathrm{R}} \sim \frac{\langle\rho \mathrm{R}\rangle}{\varepsilon^{*}} \frac{\langle\mathrm{Ov}\rangle}{\mathrm{T}^{1 / 2}} \mathrm{~m}_{0}
$$

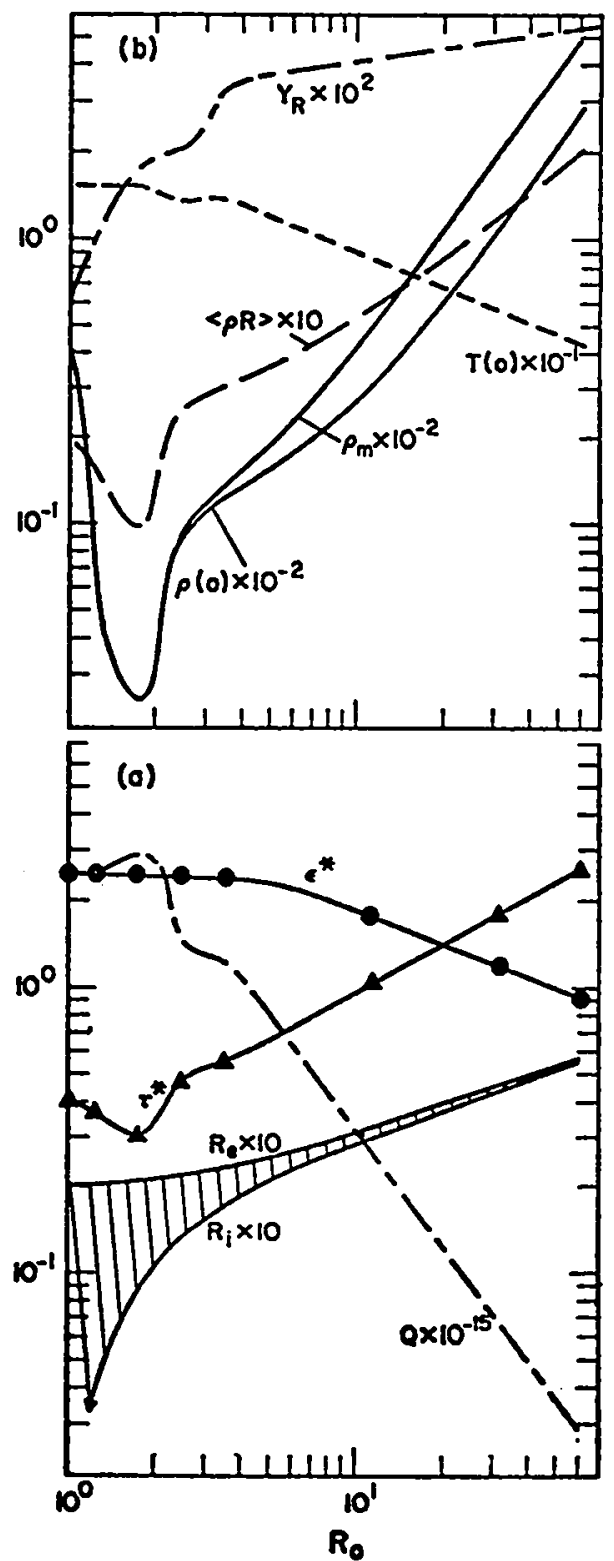

F1g. 4 Aspect ratio dependence, $p_{0}=0.213 \mathrm{~g} / \mathrm{cm}^{3}$, $\mathrm{m}=7.5 \mathrm{\mu g}$.

In the 5- to $10-\mathrm{keV}$ range $\left(\langle\sigma v\rangle / \mathrm{T}^{1 / 2}\right) \sim \mathrm{T}^{2.3}$. Thus, $X_{R} \sim R_{a}^{0.84} / R_{a}^{-0.38}\left(R_{a}^{-0.04}\right) \sim R_{a}^{0.19}$.

Indeed, the simulations give $Y_{R} \sim R_{a} 0.15$.

When $R_{a} ₹ 4$, the effects of early shell expansion compete directly with the overall compressive effects of convergence. This leads to the small $R_{a}$ excursions in $\tau^{*}$ from a smooth tuning dependence. At $R_{a}=1.75$, in particular, collapse of the inner 
shell surface yields significant pre-burn, while the main shell is still imploding, so $\langle\rho R\rangle$ and $T(0)$ alone are no longer sufficient measures of performance [as in Eq. (5)] . A higher power level is needed to drive this pre-burn condition which optimizes $Y_{R^{*}}$. Thus, $Q$ goes through a maximum.

The most noteworthy observation is that DT shells are better than DT spheres, as far as reducing $Q$ is concerned, only when $R_{a} \geqslant 2.5$. D. Mater1als Dependence

Different shell materlals may be employed for structural convenience, or to reach higher densitles. In studying volded shells of materlals other than DT, we have tuned the laser pulse parameters (in simulation) for maximum $\rho(0)$. (In the case of DT itself this requires a slight shift in the $p=1$ energy to $\varepsilon^{*}=1.27 \mathrm{~kJ}$, with $\tau^{*}=1.8 \mathrm{~ns} \operatorname{man}-$ tained.)

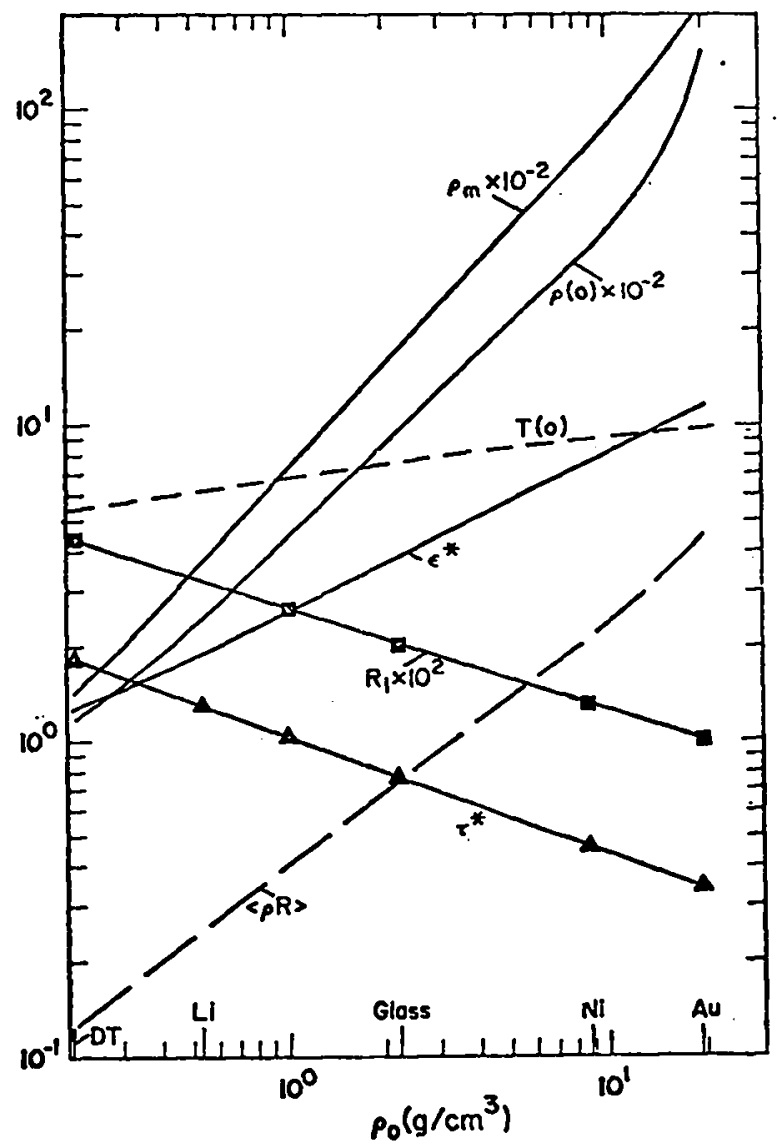

Fig. 5 Materials dependence (Initial density), m $=7.5 \mu g, R_{a}=32$.
1. Radiation "off". Figure 5 gives our 3-T code results with bremsstrahlung suppressed for $7.5 \mathrm{Hg}$ shells with $R_{a}=32$. It 18 constructed from the conditions for maximum $\rho(0)$ calculated for shells of DT, Li $\left(\rho_{0}=0.53 \mathrm{~g} / \mathrm{cm}^{3}\right), \operatorname{CDT}\left(1.0 \mathrm{~g} / \mathrm{cm}^{3}\right)$, glass $\left(2.2 \mathrm{~g} / \mathrm{cm}^{3}\right), N 1\left(8.8 \mathrm{~g} / \mathrm{cm}^{3}\right)$, and $\mathrm{Au}$ $\left(19.3 \mathrm{~g} / \mathrm{cm}^{3}\right)$.

The denser shells are smaller; $R_{i} \sim \rho_{0}^{-0.33}$. accordingly, they require a shorter pulse,

$$
\tau^{*} \sim R_{1} \sim \rho_{0}-0.33
$$

More mass is compressed with the shorter pulses: $\rho(0) \sim \rho_{0}^{0.95}$, and $\left\langle\rho R>\sim \rho_{0}^{0.76}\right.$, so by (2) $m_{0} \sim \rho_{0}^{0.37}$. Also, the central collapse temperature rises, $T(0) \sim \rho_{0}{ }^{0.13}$. These dependences explain the increased energy demands of the higher density shells, i.e., one expects

$$
\varepsilon^{*} \sim m_{0} T(0) \sim \rho_{0} 0.13 \rho_{0}^{0.37} \sim \rho_{0}^{0.50},
$$

as observed.

Higher energy and the shorter pulse increase the peak power demands on the laser: the peak power requirement of the DT shell is $1.3 \mathrm{~J} / \mathrm{ps}$, the pulse for the gold shell must terminate at $68 \mathrm{~J} / \mathrm{ps}$. The decreased size of the gold shell makes the rise in $Q$ even more marked.

The cructal observation here is that approximately a 400-fold compression applies over the full range of materials. So, by starting at a higher $\rho_{0}$, we gain access to a correspondingly higher $\rho(0)$.

2. Radiation "on". With bremsstrahlung "on" in the 3-T code ( $A$ er $\neq 0$ in Ref. 9, Eq. (B2) the DT shell performance is unchanged. The peak $\rho_{m}$ drops from $7 \times 10^{3} \mathrm{~g} / \mathrm{cm}^{3}$ to $4 \times 10^{3}$, and the $\rho_{m}$ for gold drops by more than an order of magnitude to $900 \mathrm{~g} / \mathrm{cm}^{3}$. Bremsstrahlung losses and preheat severely limit the bare gold shell compressions. About $6 \%$ of the deposited energy is radiated as bremsstrahlung from the 1ithium; $14 \%$ is so radiated from the gold shelis.

When the glass shell calculations are done with the multi-group photonics code, $\rho(0)$ drops by a factor of 5 and $\langle\rho R\rangle$ reduced 2.5 -fold. ${ }^{12}$ For the Nickel and gold shells the line- and recombination loss and pre-heat effects are even more severe. The multi-group calculations predict, ${ }^{12}$ however, that 
replacement of the outer $40 \%$ of the shell mass with low $Z$ beryllium very nearly restores the $h 1 g h$, $\mathrm{FIg}$. $5 \rho(0)$ and $\langle\rho R\rangle$ values.

\section{E. Fuel-Filled Shells}

DT fuel can be Introduced Into high $z$ shells as an 1ce-1iner or a gas fill. - One anticipates that a small mass of fuel, the order of $1 \%$ of the shell mass, should have a small effect on the shell dynamics. Thus, we start with a $7.5 \mu \mathrm{g}$ glass shell, of aspect ratio 32 , under 1 ts optimal $p=1$ tuning $\left(\varepsilon^{*}=3.8 \mathrm{~kJ}, \tau^{*}=760 \mathrm{ps}\right.$ from Fig. 5), and we calculate the consequences of adding vartous amounts of DT fuel.

1. Radiation "off". Figure 6 summarizes the 3-T results with bremsstrahlung suppressed; 6 (a) gives the pellet conditions and $Y_{R}$ obtained for different Ice-IIner masses, and versus the density of fill that would prevall if all the lce were vaporized: $6(b)$ gives the performance with a gas fill at this same density. Note that with equimolar DT, the gas density $\rho=10^{-2} \mathrm{~g} / \mathrm{cm}^{3}$ corresponds to ح 50 atm at $25^{\circ} \mathrm{C}$.

When the thermonuclear burn begins the DT fuel 1s tamped by surrounding glass. The burn character1stics of tamped systems have been treated in Ref. 11 , where we showed that

$$
\begin{aligned}
& \text { (1) }\langle\rho\rangle_{f}=\int_{0}^{R_{E}}{ }_{\rho d R} \text { and } \\
& \text { (ii) }\langle\rho R\rangle_{\text {tot }}=\langle\rho R\rangle_{E}+\int_{R_{f}}^{R_{e}}{ }_{\rho d R}
\end{aligned}
$$

are useful measures of performance. In FIg, 6 and subsequent figures these are given in $\mathrm{g} / \mathrm{cm}^{2}$.

a. DT Ice. When ice is used the maximum $X_{R}$ is calculated to be $30 \%$ when the DT mass is only $195 \mathrm{ng}$, or $2.6 \%$ of the shell mass. This optimal IIner 1s only $2.0 \mu \mathrm{m}$, or about one-third of the glass shell thickness, and about $1 \%$ of the glass Inner radius of $203 \mu \mathrm{m}$.

At the maximum $Y_{R}, T(0)=9.5 \mathrm{keV},\langle\rho R\rangle_{\text {tot }}=$ $0.5 \mathrm{~g} / \mathrm{cm}^{2}$, and $\langle\rho\rangle_{f}$, the peak average fuel density, is $340 \mathrm{~g} / \mathrm{cm}^{3}$. The corresponding conditions in the bare glass Implosion (Fig. 5) were $T(0)=7.8 \mathrm{keV}$, $\langle\rho R\rangle=0.76 \mathrm{~g} / \mathrm{cm}^{2}$ and $\rho(0)=950 \mathrm{~g} / \mathrm{cm}^{3}$. The 81 lass shell in its roll as a "pusher" brings the DT close to the central conditions achleved in the volded glass shell. The DT is compressed 1600 -fold by the pusher under the simple ramp pulse.

Decreasing the ice mass increases $T(0)$ slightly, and eventually brings $\langle\rho R\rangle_{\text {tot }}$ to the volded glass value, $0.76 \mathrm{~g} / \mathrm{cm}^{2}$, for $\mathrm{m}_{\mathrm{Dr}} \stackrel{\sim}{<} 30 \mathrm{ng}$. However, the yleld drops since there is progressively less fuel to burn.

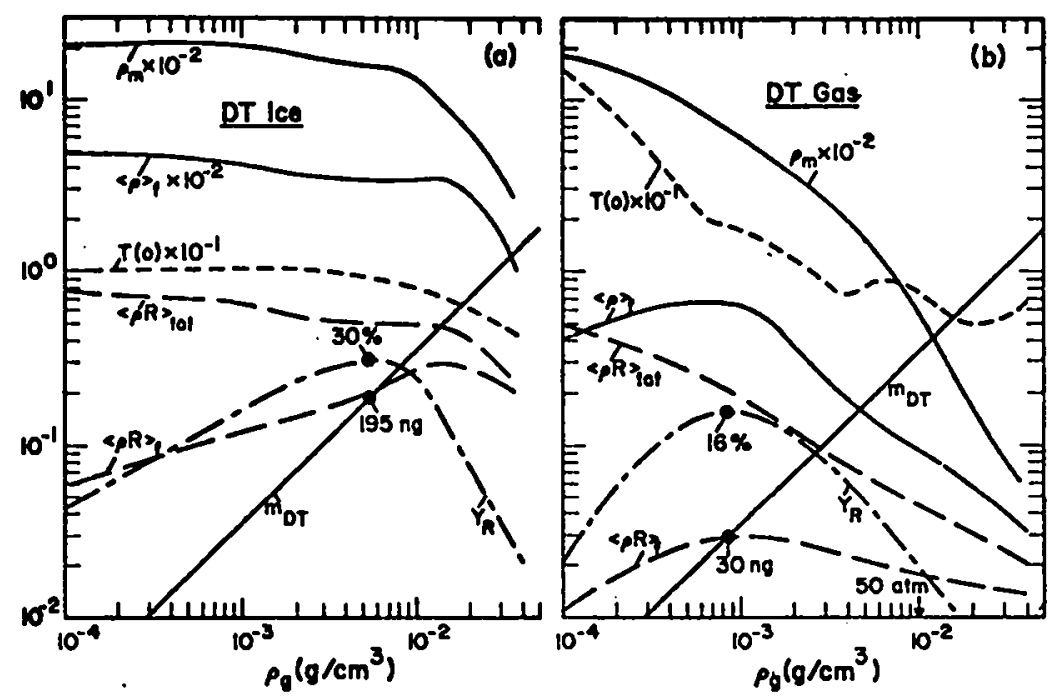

F1g. 6. Response of the $7.5-\mu \mathrm{g}, \mathrm{R}=32$, glass shell vs (a) the mass of DT ice fill, (b) the density of DT gas fill. 
Raising m $m_{D T}$ brings $\langle\rho R\rangle_{f}$ to a maximum of $0.3 \mathrm{~g} / \mathrm{cm}^{2}$ at $480 \mathrm{ng}$. But the extra DT cushions the implosion and reduces $T(0)$ to $7.5 \mathrm{keV}$, thereby depressing $\mathrm{Y}_{\mathrm{R}}$.

The optimal bare shell pulse parameters are a useful guideline, but the fuel-fllled pellet performance can be improved by additional tuning. With $\mathrm{m}_{\mathrm{DT}}$ flxed at $195 \mathrm{ng}$ and $\tau=760 \mathrm{ps}, \mathrm{Y}_{\mathrm{R}}$ is at most $39 \%$ when $\varepsilon$ is increased to $4.8 \mathrm{~kJ}$. (A further improvement in $Y_{R}$ then follows with a $\tau$ and $\varepsilon$ reduction that maintains $\varepsilon \sim \tau^{2}$.) Similarly, a $30 \%$ yleld ratio can be maintained with $m_{D T}$ as large as $950 \mathrm{ng}$, or $13 \%$ of the glass mass, if $\varepsilon$ is increased, since the large $\langle\rho R\rangle_{f}\left(>0.2 \mathrm{~g} / \mathrm{cm}^{2}\right)$ at this fuel mass can, to some extent, be traded for increased $T(0)$ so as to raise the burn rate and net output.

b. DT Gas. With gas the maximum $Y_{R}$ is $16 \%$, with only $30 \mathrm{ng}$ of fuel at $\rho_{\mathrm{g}}=9 \times 10^{-4} \mathrm{~g} / \mathrm{cm}^{3}$, which is about $5 \mathrm{~atm}$ pressure at room temperature. The gaseous fuel gets generally hotter than the corresponding mass of DT ice-liner, because it is traversed by multiple shocks ${ }^{17}$, which reflect off the inner surface of the closing glass shell, as the implosion proceeds. Hence, at maximum $Y_{R}$ :

(1) $\mathrm{T}(0)$ is $18 \mathrm{keV}$, nearly twice the ice-ltner value.

(ii) $\langle\rho R\rangle_{f} 18$ down an order of magnitude to $3 \times 10^{2} \mathrm{~g} / \mathrm{cm}^{2}$, and

(iii) $\langle\rho\rangle_{f}$ is down to $66 \mathrm{~g} / \mathrm{cm}^{3}$, although a $\rho_{\mathrm{m}}$ of $540 \mathrm{~g} / \mathrm{cm}^{3}$ is still achleved in the shel1.

When the inftial fuel density (mass) is increased, $T(0)$ and $\langle\rho R\rangle_{\text {tot }}$ drop. Backpressure from the fuel is significant earlier in the implosion sequence, and reduces the maximum compressions achievable.

With decreases in the initial fuel pressure below $5 \mathrm{~atm}, \mathrm{~T}(0)$ and $\langle\rho \mathrm{R}\rangle$ tot rise. However, the burn rate is optimal at $21 \mathrm{keV},{ }^{9}$ so, despite further $T(0)$ increases, the drop-off in $\langle\rho\rangle_{f}$ and in the burnable mass destroys $Y_{R}$. Simllarly, $\varepsilon$ Increases at the 5 atm fill pressure fall to improve the burn output.

The main points from thls examination are that: (a) considerable improvements in $\langle\rho R\rangle_{F}$ and $Y_{R}$ should be gained by freezing the DT fuel to the Inner surface of the pusher, and (b) the DT ice can be driven to as much as one-third the peak voided tamper density, or 130 times the initial tamper density, by a simple ramp pulse.

2. Radiation "on". When bremsstrahlung loss is permitted ( $A_{\text {er }} \neq 0$ ) the DT inside the microballoons radlates to significantly lower temperatures. The DT ice temperature drops from $9 \mathrm{keV}$ $\left(A_{\text {er }}=0\right)$ to $7 \mathrm{keV}$ at the 195-ng DT mass giving maximum yield. The DT gas temperature drops from about $40 \mathrm{keV}$ to about $15 \mathrm{keV}$ at $10 \mathrm{ng}$ which gives the new maximum yield. The $\langle\rho R\rangle_{f}$ and $\langle\rho R\rangle_{\text {tot }}$ curves show little change, however. The result is that the ice-filled microballoon still attains a 10-fold larger $\langle\rho R\rangle_{f}$ which should be useful in larger pellets to encourage bootstrap-heating, $9,1.1$

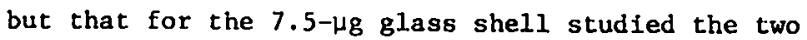
maximum yield ratios (lce vs. gas) become nearly equal at about $7 \%$. Again, the degraded $\langle\rho R\rangle$ from line- and recombination radiation can be minimized by the use of a low $\mathrm{Z}$ ablator layer.

F. The Pulse Shape

1. Gaussian Pulses. The natural output of the glass laser systems at LASL is a single Gaussian pulse, starting and terminating at about $10 \%$ of its peak power. We went through the usual tuning procedure to find the best Gaussian energy and pulse width for a 7.5- $\mu \mathrm{g}, \mathrm{R}_{\mathrm{a}}=62$, DT shell, and got the maximum yield ratio obtained with a pulse energy of $1.15 \mathrm{~kJ}$ and a full width at half-maximum of $2.07 \mathrm{~ns}$.

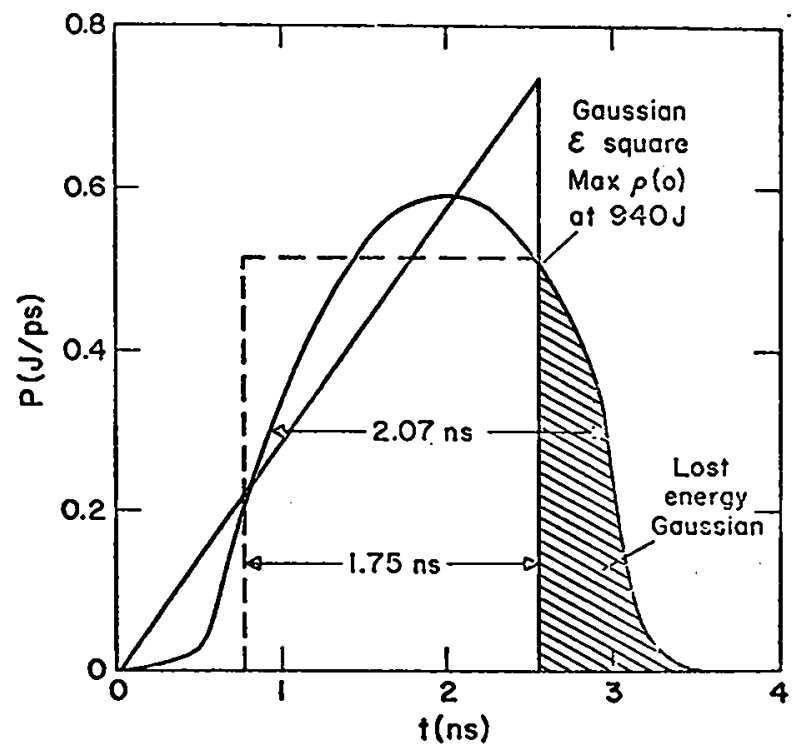

Fig. 7 Optimal $p=1$ ramp, Gaussian and square pulses for an $R_{a}=62, m=7.5 \mu g$ DT she11; the square pulsê $1 \mathrm{~s}$ delayed 800 ps. 
TARLE 4

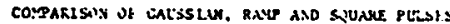

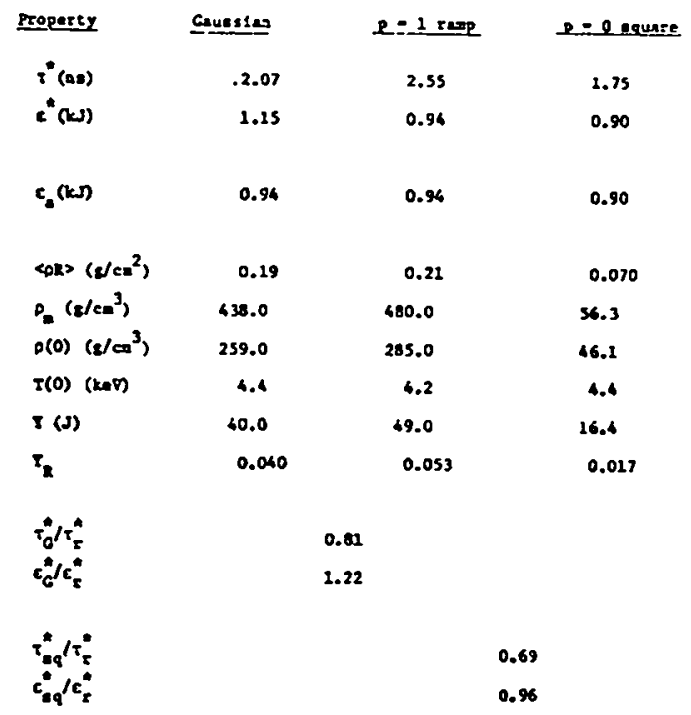

Figure 7 compares the shapes of the optimal Gaussian, and the best $p=1$ pulse. Table II records the predicted shell responses to these pulses. Note that: a) The pre-burn conditions and yield are nearly identical. b) Peak compression with the Gaussian occurs when $\varepsilon=\varepsilon^{*}=$ $9.40 \mathrm{~J}$ has been delivered; the remaining energy 18 wasted, reducing $Y_{R^{\circ}}$ c) Also, with the Gaussian a somewhat lower $\langle\rho R\rangle$ is achieved, which decreases the yield.

For correspondence, therefore, we find that the ratio of Gaussian FWhM to ramp length is $\tau_{G}^{*} / \tau_{r}^{*}=0.81$, while the ratio of energies is $\varepsilon_{G}^{*} / \varepsilon_{I}^{*}=1.23$. These ratios have been found to prevall over a broad range of $m, R_{a}$, and $\rho_{0}$ values. Thus, the optimal pulse parameters for single Gaussians follow readily from the Table I scaling rules for ramps.

2. Square Pulses $(p=0)$. Similarly, we find that

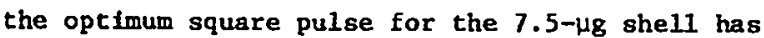
the parameters $\tau^{*}=1.75 \mathrm{~ns}$ and $\varepsilon^{*}=0.90 \mathrm{~kJ}$.

Figure 7 includes this pulse (dashed envelope), delayed $800 \mathrm{ps}$, so as to terminate in coincidence with the ramp.

Table II shows that the hydro-burn performance Is significantly reduced on going from the ramp to the square pulse. Note that $p_{m}$ drops by a factor of nine, and $\langle\rho R\rangle$ and $Y_{R}$ are down about 2.5-fold.
The connection rules between optimal square and ramp parameters are $\tau^{*}{ }_{\mathrm{sq}} / \tau_{\mathrm{r}}^{*}=0.69$ and $\varepsilon_{\mathrm{sq}}^{*} / \varepsilon_{\mathrm{r}}^{*}$. 0.96 , again, applying for a wide range of shell parameters.

3. Extended Ramps $(p>1)$. To explore the sensitivity to pulse shape we found the favored pulse parameters for a sequence of steeper ramps,

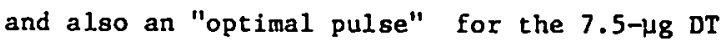
shell of $\mathrm{R}_{\mathrm{a}}=62$.

The optimal pulse form 1,2 is

$P=P_{0}\left(1-t^{\prime} / \tau\right)^{-1.875}, E\left(t_{I}\right)=\int_{0}^{t} I_{P d t}$.

We set $\tau$ to $12.5 \mathrm{~ns}$, which is suitable for DI spheres of this mass. ${ }^{10}$ Then, a large number of runs showed the best yield ratio was 5.6 for $E\left(t_{I}\right)=$ $2.5 \mathrm{~kJ}$ and $P_{0}=1.1 \times 10^{9} \mathrm{~W}$. This pulse sets $T(0)$ to $5.7 \mathrm{keV}$ just before burn (when $Y_{R}=0.01$ ), and gives $p(0)=3.04 \times 10^{3} \mathrm{~g} / \mathrm{cm}^{3}$, and $\langle\rho R\rangle=1.07$.

For the ramps, $\varepsilon^{*}$ and $\tau^{*}$ values were determined with $p$ set at $1,2,3,4,6,10,20$ and 40 . Over this range of exponents, $\langle\rho R>1$ increases from 0.21 to $0.60 \mathrm{~g} / \mathrm{cm}^{3}$. At $P=40$ bootstrap-heating 9 is encouraged by the large $\langle\rho R\rangle$, ralsing $T(0)$ from 4.4 to $7.2 \mathrm{keV}$ during the burn. This helps to increase $Y_{R}$ by a factor of 10 over its $p=1$ value.

Energy deposition $\varepsilon^{*}$ needs to be changed only slightly to maintain the optimal ramp performance. For $\mathrm{p} \ 4, \varepsilon^{*}$ asymptotes at 1.12 times its $\mathrm{p}=1$ value.

The time scale $\tau$ * changes from 2.55 to $32 \mathrm{ns.}$ This large increase is deceptive, however, as evident from Figure 8 , where selected ramps have been plotted so that they all terminate at the shutdown time of the optimal pulse. Clearly, most of the energy is delivered in the last $2 \mathrm{~ns}$, when the ramps are all quite simflar.

The ramps tend to converge to the optimal as $p$ increases: a) The peak powers rise. b) The ramp profiles move toward the optimal concavity in the interval $10 ₹ t^{\prime} ₹ 12 \mathrm{~ns}$, and c) weak prepulsing, like that of the optimal pulse, develops for $t^{\prime} ₹ 10 \mathrm{~ns}$.

We made runs to effect adjustments in the rampe In each of the separate intervals a) to c). Only the prepulse was found to be crucial to the improvement in compression. Inclusion of the $p=40, t^{\prime}=4$ to 10 ns precursor alone is sufficlent to effect the 

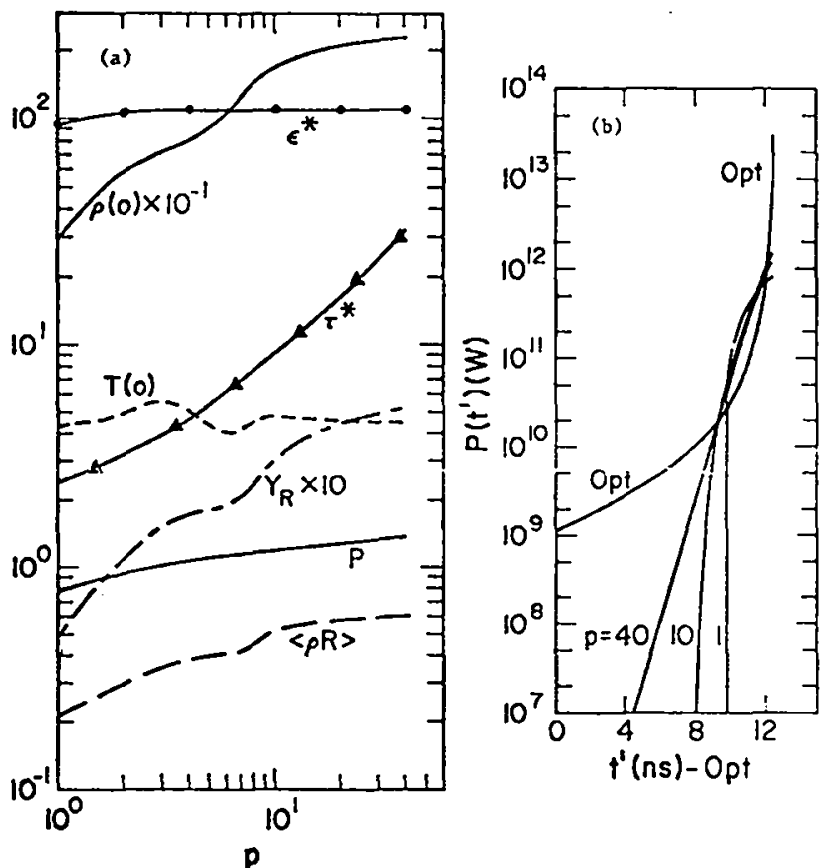

Fig. 8 (a) Performance vs the ramp exponent p,

(b) Comparison of the ramps to the fully optimal pulse: final $12.5 \mathrm{~ns}$.

three-fold increase in $\langle\rho R\rangle$. This is notable since only $3.2 \%$ of the total pulse energy arrives before $t^{\prime}=10 \mathrm{~ns}$. On the other hand, once a good compression has been achieved, the last 250 ps of the pulse should be increased (toward the "Opt" level, Fig. 8) to raise $T(0)$ from $4.4 \mathrm{keV}$ to a value that maximizes $Y_{R}$.

G. Summary

We have indicated quantitatively the improved compression and/or burn performance to expected from all of the following:

a) the use of ramps, Gaussians and square profiles at the $\varepsilon^{*}$ and $\tau^{*}$ values that match the pulses to their targets.

b) by going to pushers of higher mass, greater aspect ratio, and greater density, while retaining a low $\mathrm{Z}$ ablator.

c) with the use of DT ice, instead of gas, inside the shells, and

d) by the introduction of a weak precursor ahead of the main pulse for compression, and a i final pulse spike for temperature control.

IV. PROJECTIONS BEYOND BREAKEVEN

Concern has been expressed about the utility of structured pellets in high yield applications. ${ }^{11,} 18$ Related conjecture ${ }^{19}$ has addressed the likelihood of achieving breakeven in shellbased pellets with only one kilojoule of input energy in a simple pulse. Our calculations predict that there should be little difficulty in scaling beyond breakeven, given that the implosions are predominant $1 \mathrm{y}$ ablative and that high aspect ratio shells $\left(20 \varkappa \mathrm{R}_{\mathrm{a}} \varkappa 60\right)$ remain hydrodynamically stable. The stability question is still under theoretical 14,15,16 and experimental scrutiny.

We sought maximal $\left(A_{\mathrm{er}}=0\right)$ kilijoule response with DT ice-liner fuel as follows: We started with the F1g. $5 \mathrm{p}=1$ conditions for a glass shell of $\mathrm{m}=7.5 \mu \mathrm{g}$ and $\mathrm{R}_{\mathrm{a}}=32$; i.e., $\varepsilon^{*}=3.8 \mathrm{~kJ}$ and $\tau^{*}=$ $760 \mathrm{ps}$. Then, to reduce the energy requirements, we approached the limit of aspect ratios investigated, setting $R_{a}=59$ and getting $\varepsilon^{*}=3.0 \mathrm{~kJ}$ and $\tau^{*}=1.08 \mathrm{~ns}$ from Fig. 4. To include some pulse shaping, $p$ was then increased from 1 to 3; through Fig. 8 , this set $\varepsilon^{*}=3.8 \mathrm{~kJ}$ and $\tau^{*}=1.70 \mathrm{~ns}$. The shell mass was then reduced until. $21 \mathrm{~kJ}$ was optimal, to $\mathrm{m}=2.2 \mu \mathrm{g}$ (Fig. 3), for which $\varepsilon^{*}=1.0 \mathrm{~kJ}$ and $\tau^{*}=1.22 \mathrm{~ns}$. The time scale was lowered to $1 \mathrm{~ns}$ to

TAOLE III

TRQIECTIONS MTOND BREANEVE

\begin{tabular}{|c|c|c|c|}
\hline Casc: & $\underline{I}$ & $\underline{\underline{1}} 1$ & III \\
\hline$=z_{81}(1: 5)$ & 2.2 & 22.0 & 220.0 \\
\hline$g_{D i}(1 . \dot{\xi})$ & 0.085 & 0.85 & 8.5 \\
\hline$x_{1}(n)$ & 165.0 & 355.0 & 763.0 \\
\hline$x_{1}(1-10)$ & 166.2 & 357.5 & 768.4 \\
\hline$a_{e-1}(\mathrm{~m})$ & 169.0 & 363.6 & 781.7 \\
\hline$x_{2}$ & 59.7 & 59.4 & 59.0 \\
\hline$\tau(\mathrm{ns})$. & 1.0 & 1.86 & 3.48 \\
\hline c (a) & 1.0 & 13.7 & 188.0 \\
\hline$\langle\rho \mid\rangle_{t o t}\left(z / \dot{c}^{2}\right)$ & 0.68 & 1.63 & 2.53 \\
\hline$\langle p R\rangle_{f}\left(z / \mathrm{cr}^{2}\right)$ & 0.51 & 1.02 & 1.58 \\
\hline$\rho(0)\left(8 / \infty^{3}\right)$ & $2.5 \times 10^{3}$ & $2.3 \times 10^{3}$ & $1.4 \times 20^{3}$ \\
\hline$t(0)$ (kes) & 12.0 & 23.0 & 52.4 \\
\hline$x_{2}$ & 1.07 & 4.9 & 7.4 \\
\hline
\end{tabular}


raise the fuel temperature. Finally, we made runs for different DT ice masses until, with $85 \mathrm{ng}$, an optimum $Y_{R}$ was achieved.

Indeed, this optimum was breakeven at a kilofoule, as recorded in Table III. The required peak laser power is $4 \mathrm{~J} / \mathrm{ps}$, delfvered at an intensity of $1.1 \times 10^{15} \mathrm{~W} / \mathrm{cm}^{3}$.

Higher $Y_{R}$ from the microballoons can be obtained by simply following the Fig. 2 bare shell scaling to larger masses. We used $\varepsilon^{\star} \sim \mathrm{m}^{1.14}$ and $\tau^{\star} \sim m^{0.27}$ (Table I) with the DT and glass masses held proportional. This gives $Y_{R}=4.4$ at $\varepsilon=13.7$ $k J$ and a glass mass of $2.2 \mu g$, and $y_{R}=7.5$ with $\varepsilon=188 \mathrm{~kJ}$ for $220 \mu \mathrm{g}$ of glass (see Table III). Yleld ratios superior to the Table III values follow from Increasing the fuel mass to roughly $9 \%$ of the glass mass in larger pellets. Thus, we get $Y_{R}=$ 13 with $m_{g 1}=122 \mu g, \varepsilon=100 \mathrm{~kJ}$ and $\tau=2.9 \mathrm{~ns}$.

A. $\mathrm{A}_{\text {er }} \neq 0$. With the bremsstrahlung "on" the yeild ratio drops to only $10 \%$ at $1 \mathrm{~kJ}$, because the fuel is cooled, as in III. E.1.b. However, bootstrapheating compensates for the radiative cooling in larger pellets. Thus, we recalculate $Y_{R}=2.7$ at $14 \mathrm{~kJ}$ with the $22.0 \mu \mathrm{g}$ microsphere (II in Table III), and $Y_{R}=5.0$ with the $220 \mu g$ pellet. Breakeven thus shifts up to $3.5 \mathrm{~kJ}$, and is predicted for a $6.7 \mu g$ microballoon, of $R_{a}=59$, containing $270 \mathrm{ng}$ of DT.

\section{APPLICATION TO EXPERIMENTS}

\section{A. The LASL Experiments}

LASL has reported implosion results ${ }^{4,6}$ for glass microballoons of $27.5 \mathrm{ng}$ with an inner radius of $25 \mu \mathrm{m}$ and a wall thickness of $1.5 \mu \mathrm{m}$, i.e., of aspect ratio 17.7 .

We have extrapolated our scaling rules to determine the optimal $p=1$ ramp parameters for this target: Extension of the Fig. 3 mass curves down to $27.5 \mathrm{ng}$ gives $E=1.77 \mathrm{~J}$ and $\tau=410 \mathrm{ps}$ for $R_{a}=32$ and $\rho_{0}=0.213 \mathrm{~g} / \mathrm{cm}^{3}$ (DT). Scaling down the aspect ratio to 17.7 using FIg. 4 gives $\varepsilon=2.21 \mathrm{~J}$ and $\tau=305 \mathrm{ps}$. Finally, scaling up the density to $2.2 \mathrm{~g} / \mathrm{cm}^{3}$ with the aid of Fig. 5 gives $\varepsilon=6.1 \mathrm{~J}$ and $\tau=129 \mathrm{ps}$. An independent search for the optimum (following the III.A procedures) gives $\varepsilon^{*}=6.1 \mathrm{~J}$ and $\tau^{*}=135 \mathrm{ps}$, which is within 8\% of the tuning suggested by the scaling rules. Table IV records the calculated response of the
TABLE IV

LASL AND KMSF PELLET OPT MMAT RAMP RESPONSE

\begin{tabular}{|c|c|c|}
\hline Case & LASL & KOMSF \\
\hline${ }_{81}(08)$ & 27.5 & 26.2 \\
\hline$R_{1}$ (un) & 25.0 & 32.0 \\
\hline$\Delta R_{82}(L ⿴ 囗)$ & 1.5 & 0.9 \\
\hline . & 17.7 & 36.7 \\
\hline$\tau^{\star}(p s)$ & 135.0 & 205.0 \\
\hline$\varepsilon^{*}(J)$ & 6.1 & 5.0 \\
\hline$P(w)$ & $8.8 \times 10^{10}$ & $i .5 \times 10^{10}$ \\
\hline$Q\left(H / c n^{2}\right)$ & $1.0 \times 10^{15}$ & $5.5 \times 10^{14}$ \\
\hline$\langle\rho R\rangle\left(g / \mathrm{cm}^{2}\right)$ & $6.5 \times 10^{-2}$ & $8.0 \times 10^{-2}$ \\
\hline$D_{m}\left(g / \mathrm{cm}^{3}\right)$ & 637.0 & 966.0 \\
\hline$\rho(0)\left(g / \mathrm{cm}^{3}\right)$ & 483.0 & 830.0 \\
\hline$I(0)(k e V)$ & 2.9 & 2.0 \\
\hline
\end{tabular}

LASI shell to this optimal $p=1$ ramp. We see that $\rho(0)=483 \mathrm{~g} / \mathrm{cm}^{3}$ is predicted with $\langle\rho R\rangle=6.5 \mathrm{x}$ $10^{-2} \mathrm{~g} / \mathrm{cm}^{2}$.

The optimal single Gaussian pulse for the LASL target has a FWHM $=135 \tau_{G} / \tau_{I}=110 \mathrm{ps}$. The experiments, however, employed two 50 ps FWHM Gausslans with a variable peak-to-peak spacing of from 50 to 500 ps to allow laboratory pulse optimization. Eight percent of the ortginal pulse energy was extracted through a beam splitter; half of this was discarded, and half was delivered to the target as a prepulse. The main pulse energy $\varepsilon$ was $16 \mathrm{~J}$. A 300 ps separation $\tau_{8}$ was found experimentally to provide the best compression.

We have simulated this situation for various $\tau_{s}$ and $\varepsilon$. The results are In Fig. 9. We see that, Indeed, 300 ps is the optimum separation, giving $\rho(0)=120 \mathrm{~g} / \mathrm{cm}^{3}$ and $\langle\rho R\rangle=4 \times 10^{-2} \mathrm{~g} / \mathrm{cm}^{2}$. However, this is worse than with the optimal $110 \mathrm{ps}$ Gaussian. Figure 9 (b) shows what happens when we vary the total energy with the $4 \%$ arriving 300 ps early. The $\langle\rho R>$ achleved is very sensitive to energy over the 8 to $16 \mathrm{~J}$ range.

When the $\tau_{s}=300 \mathrm{ps}, \varepsilon=16 \mathrm{~J}$ calculation $1 \mathrm{~s}$ . run on the non-equilibrium code, ${ }^{12}$ radiative preheat 


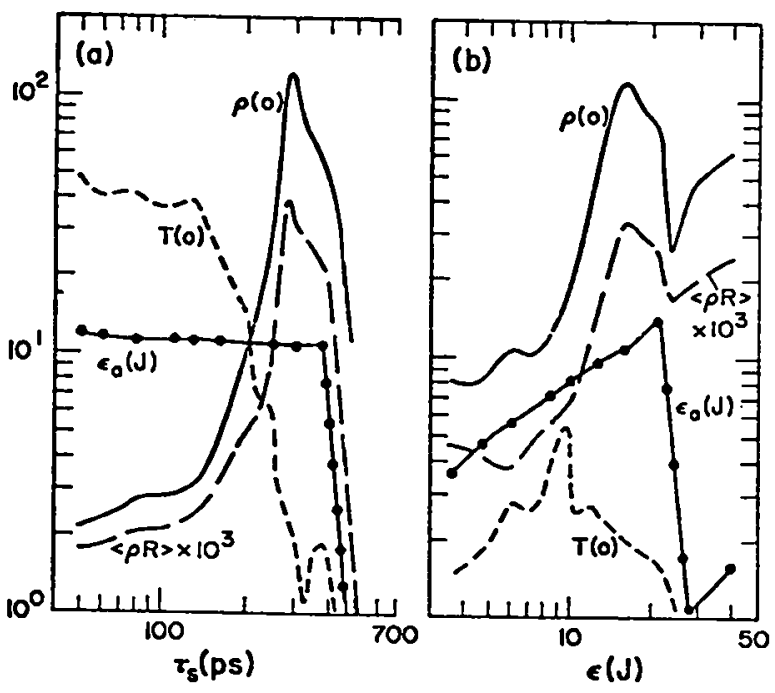

Fig. 9 Calculated performance of the LASL glass shell vs (a) spacing $\tau$ between the Gausstans, and (b) totăl energy deposition.

reduces $\rho(0)$ by a factor of five to $24 \mathrm{~g} / \mathrm{cm}^{3}$, and the maximum $\left\langle\rho R>\right.$ decreases to $1.6 \times 10^{-2} \mathrm{~g} / \mathrm{cm}^{2}$. This implies a minimum mean shell radius of $6.7 \mathrm{\mu m}$, which is consistent with the reported $10^{2}$-fold volume compression. ${ }^{4}$

\section{B. The KMSF Experiments}

KMSF has presented results from a number of gasfilled shell implosions. 5,7 we shall examine only one of these, an $R_{i}=32 \mu \mathrm{m}$ glass shell with $\Delta \mathrm{R}=$ $0.9 \mu \mathrm{m}$. Its aspect ratio was 36.7 , and 1 ts mass was $26.2 \mathrm{gg}$. The shell was filled with 18 atm of deuterium and 13 atm of tritium, corresponding to a fill density of $5.5 \times 10^{-3} \mathrm{~g} / \mathrm{cm}^{3}$ and a fuel mass of $0.75 \mathrm{ng}$. The experimental laser pulse was square $^{20}$ and 320 ps long; $8 \mathrm{~J}$ was absorbed by the target.

By extrapolating the scaling rules we find that the optimal linear ramp parameters for this shell are $\varepsilon^{*}=5 \mathrm{~J}$ and $\tau^{*}=205 \mathrm{ps}$. Table IV records our 3-T calculational result that a purely ablative implosion (i.e. with no radiative or super-thermal electron preheat) with this pulse should, indeed, yield a high peak shell density, $\rho_{\mathrm{m}}=966 \mathrm{~g} / \mathrm{cm}^{3}$, with a maximum $\langle\rho R\rangle=8.0 \times 10^{-2} \mathrm{~g} / \mathrm{cm}^{2}$. With Table II, we determine that the optimal square pulse parameters should be $\varepsilon_{\mathrm{Sq}}^{*}=(0.96) \varepsilon_{I}^{*}=4.8 \mathrm{~J}$ and $\tau_{\mathrm{sq}}^{\star}=(0.69) \tau_{\mathrm{r}}^{*}=141 \mathrm{ps}$.

A large number of 3-T simulations were run to check the square pulse response of this target.
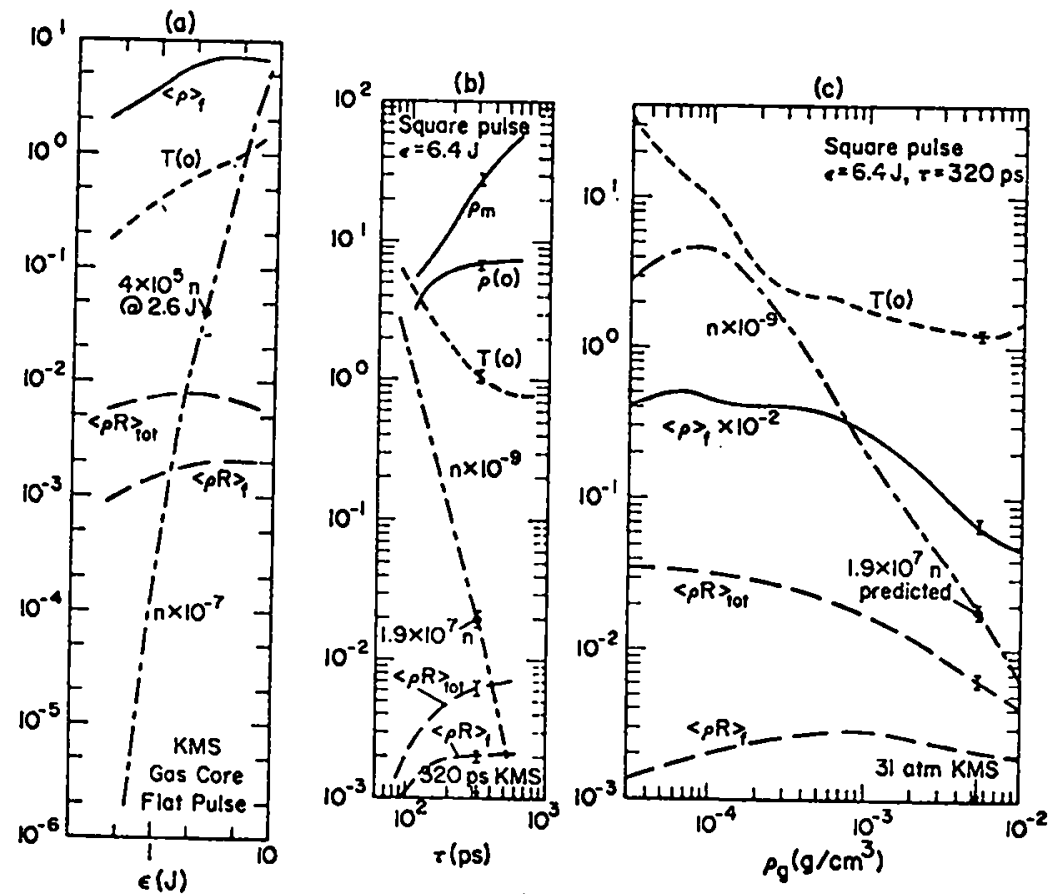

Fig. 10 Calculated response of the KMS DT gas-f 1 IIled shell vs (a) the square pulse energy $\varepsilon$, (b) the pulse time scale $\tau$, and (c) the DT gas (18-13 mix) fill density. 
Figure 10(a) shows the predictions at 31 atm for different amounts of energy absorbed over 320 p8. For (b) and (c), we varied the time scale, and fill pressure, assuming the deposition of only $6.4 \mathrm{~J}$ 1ato the classical hydrodynamics. From Fig. $10(a)$, we see that the maximum $\langle\rho R\rangle_{\text {tot }}$ and $\langle P R\rangle_{f}$ are obtained with between 3 and $8 \mathrm{~J}$, (b) shows that compression drops of $f$ rapldly for $\tau \tilde{}$ 150 ps. These results are generally consistent with the scaling rule suggestions. Figure 10 (c) shows that the neutron yield increases as the fill pressure drops. This, of course, agrees with the Fig. 6 results. The release of $1.9 \times 10^{7}$ neutrons is predicted at $31 \mathrm{~atm}$ f 111 pressure. Exper1mentally, only $4 \times 10^{5}$ neutrons were observed.

This fifty-fold discrepancy in the measured and predicted yields can be traced to a number of causes:

1. Bremsstrahlung Cooling. To reduce computation time most of our runs were made with the bremsstrahlung coupling suppressed. This has iftele effect on the compressions achleved but it overestimates the fuel temperatures, so as to overstate the neutron yleld of the KMS targets about 1.7-fold.

2. Radlative Preheat. Multi-group photonics calculations with our non-equilibrium code show that reabsorbed line- and recombination radiation photons are reducing the $\langle\rho R\rangle$ tot achieved in the microballoon implosions by a factor of 2 to 3 . This reduces the yleld below the 3-T predictions an additional 2.5-fold.

3. Two-dimenstonal Effects. 2-d calculations $^{21}$ of the implosion under up to four-fold spatial variations in the incident light intensity result in up to five-fold reductions in the yield. A similar resuit is obtained by neglecting the yield from the innermost $10 \%$ of the fuel mass, as an approximate correction for the lack of Ideal central convergence.

4. Kinetlc Effects. Henderson's results ${ }^{22}$ Indicate that the escape of fast fuel lons can lower the burn rate by about an order of magnitude at 1.5 $\mathrm{keV}$ and $\left\langle\rho R_{\mathrm{f}}=3 \times 10^{-4} \mathrm{~g} / \mathrm{cm}^{2}\right.$. For the $\left.\langle\rho R\rangle_{f}\right\rangle$ $10^{-3} \mathrm{~g} / \mathrm{cm}^{2}$ values achleved in all the Fig. $10 \mathrm{cal}-$ culations, the effect should be globally small, but on a zone to zone basis, it can be important. Below about $\mathrm{T}(0)=900 \mathrm{eV}$ the average temperature in the fuel $\langle\mathrm{T}\rangle_{\mathrm{f}}$ 1s about $70 \%$ of $\mathrm{T}(0)$. Thus, a computation of the burn based on the average temperature can lower the output by a factor of 2 or 3. Furthermore, reductions in the compression from asymetry can lower $\langle\rho R\rangle_{f}$, augmenting the fast Ion loss. Also, note that at low fill pressures in very small targets the mean-free-path for the fuel lons becomes commensurate with the dimensions of the fuel region. In this regime the lon thermal conductivity is increased, so that cooling of the fuel by contact with the glass wall is accelerated. (KMSF makes similar arguments in.Ref.23. Further, since an artifical but no real viscosity term is employed in our calculations, we undoubtedly overestimate the shock heating of the fuel under these clrcumstances.

5. Super-thermal Electron Preheat. The degree of energy deposition by long range super-thermal electrons is presently uncertatn, and possibly negligible. Here we note only that Ref. 12 showed that $4 \%$ energy deposition by super-thermals is sufficient for a < $\rho R>$ (and yield) degradation equal to that from the radiative preheat.

6. Energy Absorption. From Fig. 10(c), we see that a very slight adjustment in the assumed energy deposition can account for significant changes in the fuel temperature and the predicted neutron output.

7. Flux Lfmitation. Recent thin foil irradiation experiments at LASL have Indicated an nomalously low level of thermal conduction, suggestIng that $\beta=0.08^{13}$ may be a better choice than unity for the limiter. When $B$ is reduced to this value, the predicted neutron output drops by a factor of 1.8. The drop is closer to 10-fold, if only Inverse-bremsstrahlung absorption is allowed (no dump-all- the calculational procedure followed by KMSF ${ }^{23}$ since the rising coronal temperature with smaller $B$ reduces such absorption and hence the $T(0)$ achleved.

8. Experimental Error. The KMSF neutron measurement is stated as correct only to within a factor of two.

This collection of corrective factors is certainly sufficient to bring the optimistic 3-T code predictions into accord with the KMSF measurement. 
VI. PROBLEM AREAS

A. Prelase

In Ref. ${ }^{12}$, we showed that compression degrading radiative preheat should be substantially reduced by the use of a low $Z$ ablator layer. On the other hand, any opaque ablator materfal, such as beryllium, is likely to cause a non-negligible prelase problem, unless the overall laser system is most carefully designed. Millijoules of flash lamp light, reflected off an opaque target can cause a prepulse that totally destroys the pellet prior to the planned arrival of the main laser pulse. Thus, in early experiments one ts likely to be limited to transparent ablators such as plastic $\left(\mathrm{CH}_{2}\right)$ or frozen $\mathrm{D}_{2}$, although our calculations suggest that netther of these materials establish such favorable hydrodynamics as beryllium.

Moreover, should super-thermal electrons prove to be a problem, it may be desireable to go to higher $Z$ pushers such as nickel, so that the pusher can double as a hot electron shield. ${ }^{24}$ Here, again the potential for the opaque nickel to cause prelase may nullify this option.

B. Pulse Shaping

KMSF has recently completed a set of linear ramp pulse experfments, as part of its contract with ERDA. ${ }^{23}$ The targets were $R_{a} \simeq 40$ microballoons, like the one described by Fig. 10, but filled with efther 10 or 100 atm of DT. The laser pulse was $60 \mathrm{~J}$, delivered in $480 \mathrm{ps}$. Typically, $5 \mathrm{~J}$ was absorbed. No neutrons were detected in these experiments; the threshold for neutron detection was about $10^{3}$. The pinhole pictures regularly showed 4-fold asymetry feacures, indicative of shell break-up. The inner shell radius $R_{1}$ converged no further than to $R_{i} / 3$ under the ramps, while convergence to $R_{i} / 5$ or $R_{i} / 10$ was accomplished with square pulses. The $5 \mathrm{~J}$ is only $60 \%$ of the energy absorbed with the square pulses. We have learned, ${ }^{20}$ moreover, that neutrons have never been seen in ramp pulse experiments by KMSF, all of these being for $\tau>400 \mathrm{ps}$, and most being a part of a much earlier experimental series, for which $200 \mathrm{~J}$, or more, was delivered by the laser.

Our simulations tell us that, with fixed total energy deposition, the ramps should yield twice as many neutrons as square pulses of equal length. However, under a $60 \%$ reduction in input energy, the predicted neutron count can drop by more than two orders of magnitude [as in F1g. 10 (a), below the $5 \times 10^{4}$ n point]. If, then, one also takes Into account the observed reduced compression, and consequent kinetic effects, the loss of neutrons with ramps is understandable.

On the other hand, pulse shaping is most desireable for high compression, as discussed in Sec. III. F. The lack of success with ramp pulses is, therefore, troublesome. In the KMSF-ERDA shots the ramp pulses were more than 2.5 times too long, 1.e., $480 \mathrm{ps}$, while the optimum $\tau^{*}$ should be closer to 205 ps [see Table IV]. Experiments will be needed to determine if the Instability problems are reduced as $\tau \rightarrow \tau^{*}$.

KMSF argues ${ }^{23}$ that under the 480 ps ramps the exterior of the microballoons remains cool too long to accomodate instability suppression by transverse conduction. This situation should be alleviated, in part, by: (a) preheating the shell exterior by means of a flash prepulse ${ }^{16}$ at the start of the ramp, (b) again, through the use of a high conductivity, low $\mathrm{z}$ ablator coating on the microballoons, and (c) posstbly through the use of longer initial wavelengths, e.g. $10.6 \mu \mathrm{m}$, since they naturally lead to higher temperatures at the critical surface.

\section{Absorbed Energy}

Good target performance requires effective energy absorption [F1gs. 2(b) and 10(b)], and absorption at high terminal intensities is needed for strong final shocks and high central fuel temperatures [Sec. III.F]. of $60 \mathrm{~J}$ in a square pulse from their mirror-lense system KMSP workers find that $80 \%(48 \mathrm{~J})$ is delivered to the target area; $33 \%$ of this $(16 \mathrm{~J})$ is unrefracted, of which $50 \%(8 \mathrm{~J})$ is typically absorbed. This indicates a need for modified optics, providing more nearly perpendicular delivery of the light rays to the spherical microballoons.

Inverse-bremsstrahlung is an efficient absorption mechanism in glass at currect experimental intensities $\left(\approx 10^{15} \mathrm{~W} / \mathrm{cm}^{2}\right)$. As we go to low $\mathrm{Z}$ ablator layers, however, its effectiveness is reduced. More of the energy may absorb anomalously, or simply reflect. A solution is to frequency 
double or triple the $1.06 \mu$ light, but presently only 20 to $30 \%$ of the laser energy can be converted to the shorter wavelengths.

More complicated target geometrles, such as the LASL "ball and disk", 4 are aimed at enhancing absorption efficiency at the requisite intensities. D. Cryogenics

In Sec. III.E, we described the theoretical advantages of imploding DT ice-lined shells. Two practical problems with such targets should be mentioned:

(1) The rapid $\beta^{-}$decay of the tritium to form $\mathrm{He}^{3}$ results in considerable self-heating of the DT. Thus, unusual experimental haste may be demanded. Any lengthy interlude between the mountIng and shooting of a target may lead to the evolution of troublesome background gas. Figures 6 (a) and 10 (c) showed that even one atm of DT gas must be considered to have a significant effect on the implosive hydrodynamics.

(2) More limiting, at present, is the fact that direct atcempes to freeze DT in the microballoons result in the formation of a localized DT globule in the balloon, rather than the desired, uniform DT ice-liner. A cure may be to implode the pellet while the DT is in a transient liquid state. When the DT globule is heated by, say, a flash-lamp, it spreads somewhat smoothly over the full inner surface of the microballoon, and then evaporates.

\section{ACKNOWLEDGMENTS}

The author is indebted to R.L. Morse for many helpful suggestions, to W. P. Gula for assistance in carrying out the computer calculations, and to G. S. Fraley for the computations from his non-equilibrium hydro-burn code. ${ }^{9}$

\section{REFERENCES}

1. J. Nucko1ls, I. Wood, A. Theissen, and Zimmerman, "Laser Compression of Matter to Super-High Densities: Thermonuclear (CTR) Applications," Nature 239, 139 (1972).

2. J. S. Clarke, H. N. Fisher, and R.J. Mason, "Laser-Driven Implosion of Spherical DT Targets to Thermonuclear Burn Conditions," Phys. Rev. Lett. 30, 89 (1973), and 30, 249 (1973).

3. K. Brueckner, "Laser-Driven Fus1on," IEEE Transactions on Plasma Sciences PS-1, (1973), and $K$. Brueckner and S. Jorna, "Laser-Driven
Fusion," Rev. Mod. Phys. 46, 325 (1974).

4. G.H. McCall and R. L. Morse, "Target Compression with One Beam," Laser Focus 10, 40 (December) 1974, and D. B. Henderson, "Theoretical and Practical Aspects of Laser Fusion," Bull. Am. Phys. Soc. 19, 886 (1974).

5. R. R. Johnson, "Observations of D-T Neutron Production from Laser Driven Implosion," Bull. Am. Phys. Soc. 19, 867 (1974).

6. R. F. Benjamin, K. Boyer, E. H. Farnum, C. A. Fenstermacher, R. J. Fries, D. V. Glovanielli, R. P. Godwin, A. J. Lieber, G. H. McCall, and T. F. Stratton, "Carbon Dloxide Laser Development and Asymetry in Laser-Driven Implosions," Fifth I.A.E.A. Conference on Plasma Physics and Controlled Thermonuclear Research, Tokyo, Japan, Nov. 11-15, 1974, paper IAEA-CN33/F4-5.

7. G. Charatis, Downward, R. Goforth, B. Guscott, T. Henderson, S. Hildum, R. Johnson, K. Moncur, T. Leonard, F. Mayer, S. Segall, L. Slebert, D. Solomon, and C. Thomas, "Experimental Study of Laser Heated Spherical Targets," Fifth I.A.E.A. Conference on Plasma Physica and Controlled Thermonuclear Research, Tokyo, Japan, Nov. 11-15, 1974, paper IAEA-CN-33/F1.

8. P. M. Cambell, G. Charatis, and G. P. Montry, "Laser-Driven Compression of Glass Microspheres," Phys. Rev. Lett., 34, 74 (1975).

9. G. S. Fraley, E. J. Linnebur, R. J. Mason, and R. L. Morse, "Thermonuclear Burn Characteristics of Compressed Deuterium-Tritium Microspheres," Phys. Fluids 17, 474 (1974).

10. R.J. Mason and R. L. Morse, "Kydrodynamics and Burn of Optimally Imploded Deuterium-Tritlum Spheres," Phys. Fluids 18, 816 (1975).

11. R. J. Mason and R. L. Morse, "Tamped Thermonuclear Burn of DT Microspheres," (Submitted to Nuclear Fusion - Letters).

12. G. S.Fraley and R. J. Mason, "Preheat Effects on Microballoon Laser Fusion Implosions," (submitted to Phys. Rev. Lett.).

13. R. C. Malone, R. L. McCrory, and R. L. Morse, "Indications of Strongly Flux-1imited Electron Thermal Conduction in Laser-Target Experiments," Phys. Rev. Lett. 34, 721 (1975).

14. J. N. Shiau, E. B. Goldman, and C. I. Weng, "Linear Stability Analysis of Laser-Driven Spherical Implosions," Phys. Rev. Lett. 32, 352 (1974).

15. G. S. Fraley, W. P. Gula, D. B. Henderson, R. L. McCrory, R. J. Mason, and R. L. Morse, "Implosion, Stability and Burn of Multishell Fusion Targets," Fifth I.A.E.A. Conference on Plasma and Controlled Thermonuclear Research, Tokyo, Japan, Nov. 11-15, 1974, paper IAEACN-33/F 5-5.

16. J. Nuckolls, J. Iindl, W. Mead, A. Thlessen, 
L. Wood, and G. Zimmerman, "Laser Driven Implosion of Hollow Pellets," Fifth I.A.E.A. Conference on Plasma Physics and Controlled Thermonuclear Research, Tokyo, Japan, Nov. 11-15, 1974, paper IAEA-CN-33/F 3-4.

17. C. Evans and F.' J. Ėvans, "Shock Compression of a Perfect Gas," Fluld Mech. ㄱ, 399 (1956).

18. J. Nuckolls and Y. L. Pan, "Gain of Laser Fusion Pellets," Bull. Am. Phys. Soc. 19, 949 (1974).

19. "News and Opinion," Physics Today, August 1972, p. 17.

20. R. Johnson, KMSF, private communication, Apr11 10, 1975.
21. J. Nuckolls and R. Theissen, LLL, private communication, April 4, 1975.

22. D. B. Henderson, "Burn Characterist1cs of Marginal Deuterium-Tritium Microspheres," Phys. Rev. Lett. 33, 1142 (1974).

23. KMS Fusion Report U297, Feb. 28, 1975 (unpublished), KMS Fusion Data Report on contract (49-1)-3758 Part I, March 3, 1975 (unpublished), and KMS Fusion Report U323, Apri1 12, 1975 (unpublished).

24. R. L. Morse and C. W. Nielson, "Occurence of High Energy Electrons and Surface Expansion in Laser Heated Plasmas," Phys. Flulds 16, 909 (1973), see p. 912.

\section{APPENDIX A}

\section{CODE FEATURES}

Tapered zoning was employed to improve calculational efficiency. Thus, the zones were finest at the surfaces of the pusher and fuel shells, and at the edge and center of the gas cores. The pusher-tamper surface zones were a fraction $\Delta R_{\text {m }} / \Delta R=$ 0.005 of the total shell width. The gas and ice fuel zones were a fraction $\Delta R_{m} / \Delta R=0.05$ of the 1ce fuel-shell width, or of the gas fuel sphere radius. In both the pusher and fuel regions the zone sizes increased from the Inner surface or center by the ratio $\Delta R_{m+1} / \Delta R_{m}=1.2$, and decreased from the outer surfaces by the ratio $\Delta R_{m-1} / \Delta R_{m}=0.80$. This generated 32 shell zones in all, and 12 fuel zones in the filled systems.

Finer zoning was avolded to mintmize running time, which was typically 2.5 minutes of $\operatorname{CDC} 7600$
CPU time per implosion. Predictions with finer zoning are generally more optimistic, i.e., higher $\rho(0), \rho_{m}$ and $T(0)$. Ultimately, a proper choice for the minimum zone size in one-dimensional calculations must cone from two or three dimensional considerations which set the maximum degree of central convergence actually attainable in the laboratory.

One approximation should be noted: In the shell implosions, the kinetic energy of the innermost zone was discarded upon the zone's first contact with the center. The inner zone boundary was subsequently tied to the center. This decreases $T(0)$ and raises $p(0)$ somewhat upon the later final shock collapse; however, $\rho_{\mathrm{m}}$ remains essentially unchanged.

APPENDIX B

\section{BREMSSTRAHLUNG PREHEAT}

For the bremsstrahlung results reported in the main text we used fully ionized 2 values in the electron-radiation coupling coefficient $A_{e r}$ of Ref. 9. More recently, we have determined that with the proper partially ionfzed 2 values in $A_{e r}$ the calculated radiative preheat in glass increases significantly, approaching the levels reported from our multi-group photonics calculations of Ref. 12 . With partial ionization inverse-bremsstrahlung absorption in the cold glass below the ablation surface is decreased by nearly an order of magnitude. Thus, bremsstrahlung penetrates more deeply prior to coupling to the electrons, typically reducing the glass pusher-tamper density by a factor of three below the values calculated for full ionization. 